\title{
STABILITY OF ELASTICALLY RESTRAINED VALVE-PIPE SYSTEM WITH CRACK
}

\author{
KWAN DO HUR, IN SOO SON ${ }^{\dagger}$ and SEONG CHUL LEE \\ Department of Mechanical Engineering, Dong-eui University, 995 Eomgwangno, Busanjin-Gu, \\ Busan, 614-714, South Korea \\ †isson92@deu.ac.kr
}

\begin{abstract}
The dynamic stability and natural frequency of elastically restrained pipe conveying fluid with the attached mass and crack are investigated in this paper. The pipe system with a crack is modeled by using extended Hamilton's principle with consideration of bending energy. The crack on the pipe system is represented by a local flexibility matrix and two undamaged beam segments are connected. The crack is assumed to be in the first mode of fracture and to be always opened during the vibrations. From the governing equations, the influence of attached mass, its position and crack on the dynamic stability of elastically restrained pipe system is presented. Also, the critical flow velocity for the flutter and divergence due to the variation in the position and stiffness of supported spring is studied. Finally, the critical flow velocities and stability maps of the cracked pipe conveying fluid with the attached mass are obtained by the changing parameters.
\end{abstract}

Keywords: Stability; Flutter; Pipe Conveying Fluid; Open Crack; Critical Flow Velocity.

\section{Introduction}

Flow-induced instability of the pipe conveying fluid has attracted much scientific interest [1]. When pipes are subjected to damage, its stability is varied due to the change of its mechanical characteristics [2]. Therefore, it is very important to study the coupling effect between the fluid and crack. The dynamic stability of cracked pipes conveying fluid has been studied for a long time. It has established that a straight cantilevered pipe is stable for flow velocities less than a certain value, called the critical flow velocity. If such a pipe conveys fluid has the critical flow velocity, it loses stability by flutter [3]. Liu et al.[4] examined the suitability of using coupled response to detect damage in thin-walled tubular structures. Dado and Abuzeid [5] studied modeling and analysis algorithm for cracked beams by considering the coupling between the bending and axial modes of vibration. Kang [6] investigated the effect of rotary inertia of concentrated masses on the instability of a pipe conveying fluid. Recently, reviews on vibration of cracked structures were reported by Wauer [7] and Dimarogonas [8], and many researchers investigated the dynamic stability of a pipe conveying fluid with crack [9-11]. However, other researches did not study about the coupling effects of an attached mass, fluid flow, spring support 
and crack for stability analysis of pipe system. Therefore, in this paper, the effects of the mass ratio, the spring constant, a crack and attached mass on the stability and instability of valve-pipe system are studied by numerical method.

\section{Mathematical Model}

Figure 1(a) shows the schematic diagram of an elastically restrained pipe conveying fluid with an attached mass and crack. Where $L$ is the total length of pipe, $m_{v}$ is an attached mass, $U$ and $x_{c}$ are the velocity of fluid flow and the position of a crack, respectively. The pipe is resting on an intermediate spring support located at a distance $x_{k}$ from the left end of the pipe. The spring support is assumed to be linear with spring constant $K$. Figure 1(b) shows a circular hollow cross-section of the cracked section. $\theta_{c}$ and $2 b$ are the half-angle of the total crack depth(the crack severity will be indicated by $\alpha=\theta_{c} / \pi$ as percentage) and the length of a crack, respectively.

\subsection{Crack modeling}

The additional strain energy due to the crack can be considered in the form of a flexibility coefficient expressed in terms of the stress intensity factor, which can be derived by Castigliano's theorem in the linear elastic range. Therefore, the local flexibility in the presence of the width $2 b$ of a crack is defined by [2]

$$
C_{i j}=\frac{\partial u_{i j}}{\partial P_{j}}=\frac{\partial^{2}}{\partial P_{i} \partial P_{j}}\left(\int_{-b}^{b} \int_{0}^{a_{c}} J d y d z\right),
$$

where $P_{i}$ and $u_{i j}$ are the load in the same direction as the displacement and a local additional displacement, respectively[4]. The strain energy density function $J$ is

$$
J=\frac{1}{E^{*}}\left(K_{I M}\right)^{2}
$$

where $E^{*}=E /\left(1-v^{2}\right)$ for the plane strain, $E$ and $v$ are Young's modulus and Poisson's
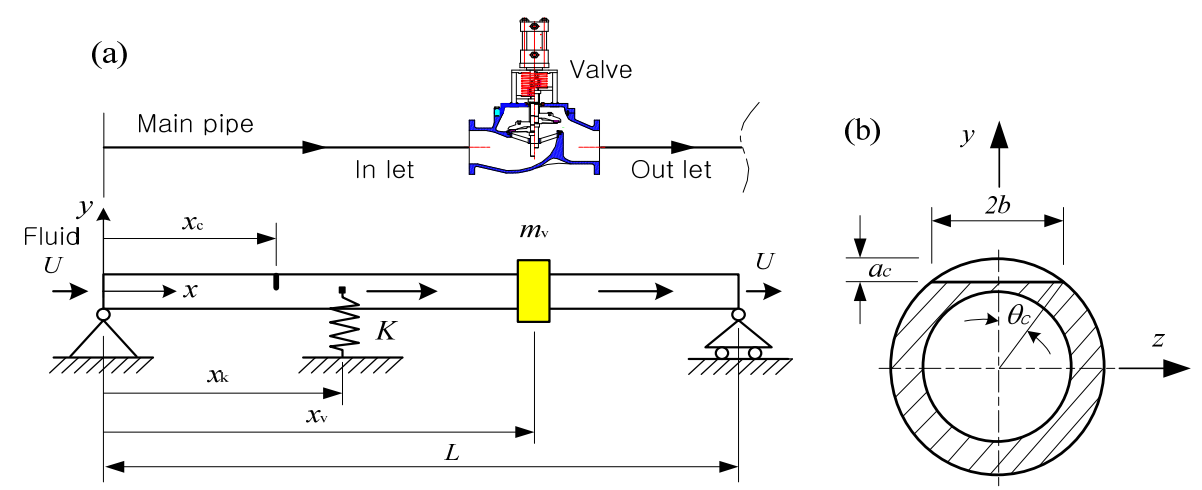

Fig. 1. (a)Geometry of analytical model and (b)Cross-section of cracked pipe 
ratio, respectively. The stress intensity factors of bending is given by

$$
K_{I M}=\frac{M_{b}}{\pi R^{2} t_{p}} \sqrt{\pi R \theta_{c}} F_{b}\left(\theta_{c}\right)
$$

where $R$ is the mean radius, $M_{b}$ and $t_{p}$ denote bending moment and the thickness of pipe. The function $F_{b}\left(\theta_{c}\right)$ is given in reference [4]. The crack stiffness used in this study are defined as

$$
K_{c} \equiv C_{i j}^{-1}
$$

\subsection{Dimensionless equation of motion}

The equations of motion of the present modeling are obtained by the application the Hamilton's principle. For simplicity, the following dimensionless quantities are introduced:

$$
\begin{aligned}
& u=L U \sqrt{\frac{M}{E I}}, \xi_{v}=\frac{x_{v}}{L}, \beta=\frac{M}{M+m}, \mu=\frac{m_{v}}{(M+m) L}, \alpha=\frac{\theta_{c}}{\pi}, \\
& \xi=\frac{x}{L}, \quad \xi_{k}=\frac{x_{k}}{L}, \quad \xi_{c}=\frac{x_{c}}{L}, \quad k=\frac{K L^{3}}{E I}, \tau=\frac{t}{L^{2}} \sqrt{\frac{E I}{M+m}}
\end{aligned}
$$

where $m$ and $M$ are the mass of pipe and fluid per unit length and $\beta$ and $E I$ denote the relative mass of fluid and the flexural rigidity of the pipe, respectively. $\mu$ and $\alpha$ are the relative mass of the attached mass and crack severity. The dimensionless quantity of $y(x, t)$ is given by

$$
\eta_{n}(\xi, \tau)=\frac{y_{n}}{L}=Y_{n}(\xi) e^{i \omega \tau} ;(n=1,2),
$$

where $n$ is the number of the segments due to the crack. In this study, we consider only the bending vibration. Therefore, the dimensionless equations of motion for bending vibration of the cracked pipe conveying fluid with an attached mass are obtained as follows:

(i) $0 \leq \xi_{v} \leq \xi_{c}$;

$$
\text { if } \begin{aligned}
& 0 \leq \xi_{k} \leq \xi_{c} \\
& \eta_{1}^{i v}+u^{2} \eta_{1}^{\prime \prime}+2 u \sqrt{\beta} \dot{\eta}_{1}^{\prime}+k \delta\left(\xi-\xi_{k}\right) \eta_{1}+\left\{1+\mu \delta\left(\xi-\xi_{v}\right)\right\} \ddot{\eta}_{1}=0, \quad\left(0 \leq \xi \leq \xi_{c}\right) ; \\
& \eta_{2}^{i v}+u^{2} \eta_{2}^{\prime \prime}+2 u \sqrt{\beta} \ddot{\eta}_{2}^{\prime}+\ddot{\eta}_{2}=0, \quad\left(\xi_{c} \leq \xi \leq 1\right),
\end{aligned}
$$

else,

$$
\begin{aligned}
& \eta_{1}^{i v}+u^{2} \eta_{1}^{\prime \prime}+2 u \sqrt{\beta} \dot{\eta}_{1}^{\prime}+\left\{1+\mu \delta\left(\xi-\xi_{v}\right)\right\} \ddot{\eta}_{1}=0, \quad\left(0 \leq \xi \leq \xi_{c}\right) \\
& \eta_{2}^{i v}+u^{2} \eta_{2}^{\prime \prime}+2 u \sqrt{\beta} \dot{\eta}_{2}^{\prime}+k \delta\left(\xi-\xi_{k}\right) \eta_{2}+\ddot{\eta}_{2}=0, \quad\left(\xi_{c} \leq \xi \leq 1\right)
\end{aligned}
$$

(ii) $\xi_{c} \leq \xi_{v} \leq 1$; 


$$
\begin{aligned}
& \text { if } 0 \leq \xi_{k} \leq \xi_{c} \\
& \eta_{1}^{i v}+u^{2} \eta_{1}^{\prime \prime}+2 u \sqrt{\beta} \dot{\eta}_{1}^{\prime}+k \delta\left(\xi-\xi_{k}\right) \eta_{1}+\ddot{\eta}_{1}=0, \quad\left(0 \leq \xi \leq \xi_{c}\right) ; \\
& \eta_{2}^{i v}+u^{2} \eta_{2}^{\prime \prime}+2 u \sqrt{\beta} \dot{\eta}_{2}^{\prime}+\left\{1+\mu \delta\left(\xi-\xi_{v}\right)\right\} \ddot{\eta}_{2}=0, \quad\left(\xi_{c} \leq \xi \leq 1\right) ;
\end{aligned}
$$

else

$$
\begin{aligned}
& \eta_{1}^{i v}+u^{2} \eta_{1}^{\prime \prime}+2 u \sqrt{\beta} \dot{\eta}_{1}^{\prime}+\ddot{\eta}_{1}=0, \quad\left(0 \leq \xi \leq \xi_{c}\right) ; \\
& \eta_{2}^{i v}+u^{2} \eta_{2}^{\prime \prime}+2 u \sqrt{\beta} \dot{\eta}_{2}^{\prime}+k \delta\left(\xi-\xi_{k}\right) \eta_{2}+\left\{1+\mu \delta\left(\xi-\xi_{v}\right)\right\} \ddot{\eta}_{2}=0, \quad\left(\xi_{c} \leq \xi \leq 1\right) ;
\end{aligned}
$$

where $(\cdot)$ is the dimensionless time derivative, and ( )' stands for partial differentiation with respect to $\xi$. $\delta$ denotes Dirac delta function. The boundary conditions are obtained in Ref. [2]. By using the equations of motion, it can obtain the complex eigenvalue $\omega$. The stability of the cracked valve-pipe system is determined by the sign of imaginary part of $\omega$. As the flow velocity increases, the imaginary part of $\omega$ becomes negative, and the pipe system loses stability by buckling for $\operatorname{Re}(\omega)=0$ and by flutter for $\operatorname{Re}(\omega) \neq 0$. If $\operatorname{Im}($ $\omega)=0$, it has the critical flow velocity $u_{c r}$.

\section{Numerical Results and Discussion}

In this study, the dynamic stability of an elastically restrained valve-pipe conveying fluid with crack is investigated.

Figure 2 shows the map of different kinds of instabilities for valve-pipe system with $\xi_{v}=0.4, \xi_{k}=0.5$ and $k=100$. The horizontal axis is the mass ratio and the axis of the ordinates is the fluid velocity. In Figure 2(a), as the crack increases, the region of flutter instability of pipe system is decreased. In Figure 2(b), when the crack position and mass ratio are the same as 0.2 , as the fluid velocity increase, the pipe system is changed as 'stable $->$ divergence $->$ flutter $->$ divergence'.

The effects of spring constant on the dynamic stability of pipe system are shown in Figure 3 , where $\beta=0.4, \xi_{v}=0.4$ and $\xi_{k}=0.5$. When the crack severity is constant, the spring constant is proportional to the stable region of pipe system. When the crack

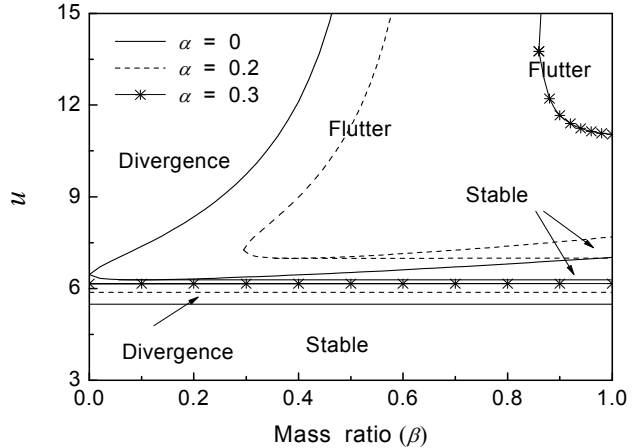

(a)Effect of crack depth $\left(\xi_{c}=0.5\right)$

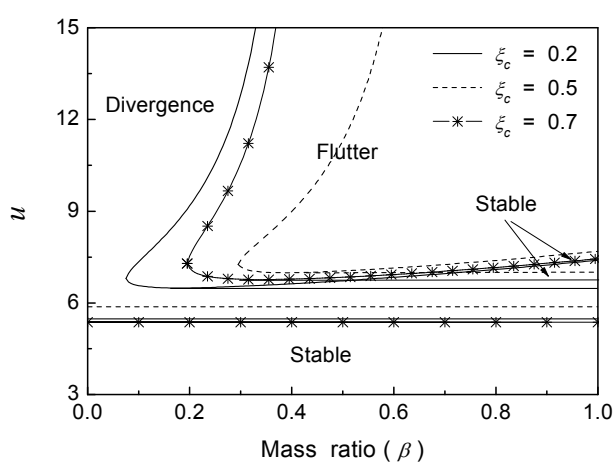

(b)Effect of crack position $(\alpha=0.2)$

Fig. 2. Map of different kinds of instabilities for pipe conveying fluid( $\xi_{v}=0.4, \xi_{k}=0.5$ and $k=100$ ) 
position exists in the both ends of pipe, the unstable region for flutter is increased.

Figure 4 presents the map of different kinds of instability for pipe system with $\beta=0.4, \xi_{v}=0.4$ and $k=100$, respectively. The horizontal axis is the spring position and the axis of the ordinates is the fluid velocity. As the crack severity increase, the stable region of pipe system is increased in the ranges $0.10<\xi_{k}<0.85$. When the crack severity is 0.3 , the pipe system has not the unstable region for flutter in the ranges $0.28<\xi_{k}<0.77$. In Figure 4(b), it is noted that the phenomenon of jump take place at the crack position. Using this phenomenon, it can be possible the detection of crack in pipe system.

Figure 5 shows the stability map of cracked valve-pipe system with $\beta=0.4, \xi_{k}=0.4$ and $k=100$. The horizontal axis is the position of attached mass. When the crack severity is 0.3 , the critical flow velocity $u_{c r}$ has the smallest value at $\xi_{v}=0.78$. If $\alpha=0$, the pipe system has the re-stable region in any position of attached mass. In Figure 5(b), the pipe system has the re-stable region in the ranges $0<\xi_{v}<0.20$ and $0.41<\xi_{v}<0.87$. If the attached mass exists in the center of the pipe conveying fluid, the divergence unstable region appears as the fluid velocity increase.

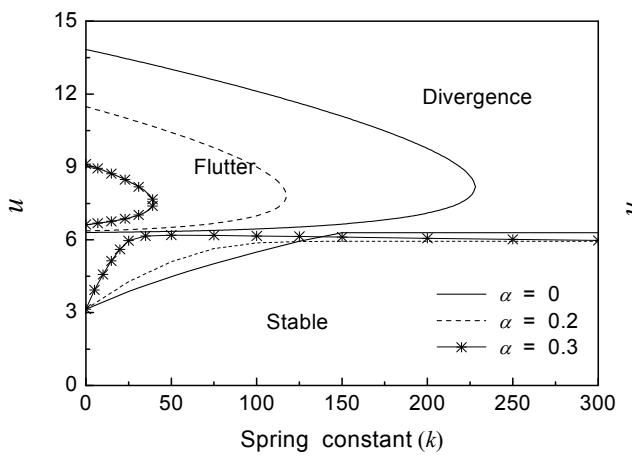

(a)Effect of crack depth $\left(\xi_{c}=0.5\right)$

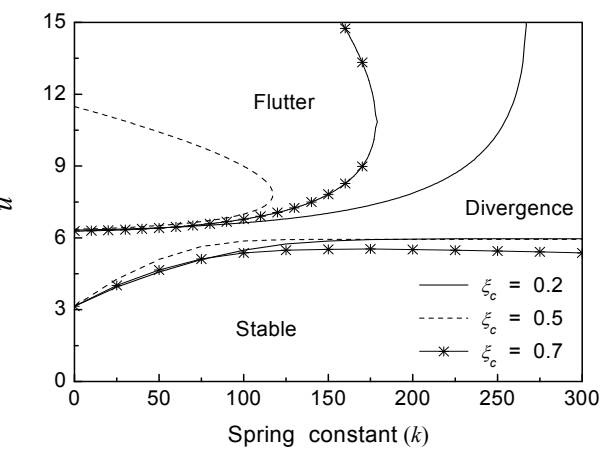

(b)Effect of crack position $(\alpha=0.2)$

Fig. 3. Map of different kinds of instabilities for pipe conveying fluid $\left(\beta=0.4, \xi_{v}=0.4\right.$ and $\left.\xi_{k}=0.5\right)$

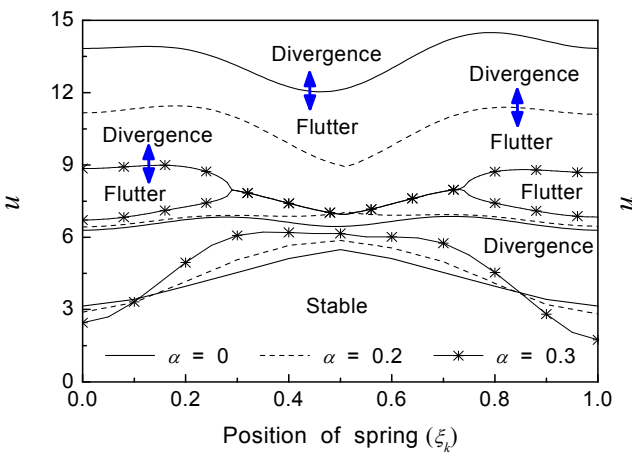

(a)Effect of crack depth( $\left.\xi_{c}=0.5\right)$

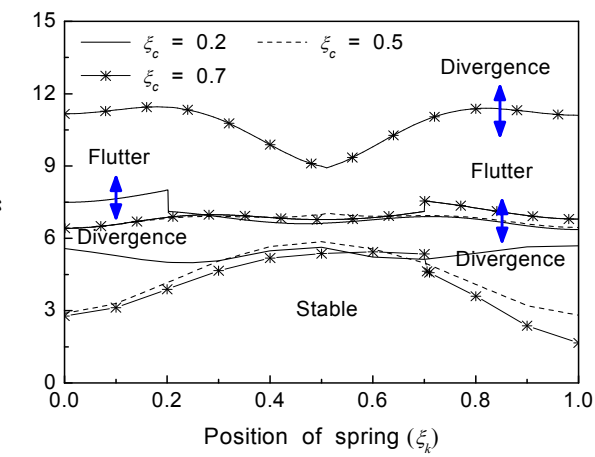

(b)Effect of crack position $(\alpha=0.2)$

Fig. 4. Map of different kinds of instabilities for pipe conveying fluid $\left(\beta=0.4, \xi_{v}=0.4\right.$ and $\left.k=100\right)$ 


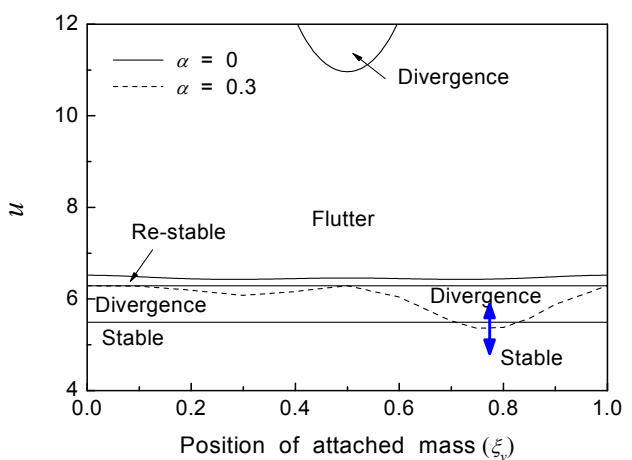

(a) $\alpha=0,0.3$

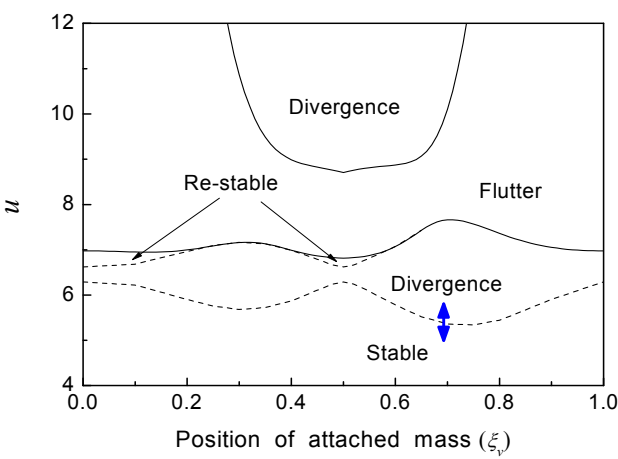

(b) $\alpha=0.2$

Fig. 5. Map of different kinds of instabilities for pipe conveying fluid $\left(\beta=0.4, \xi_{k}=0.4, \xi_{c}=0.5\right.$ and $\left.k=100\right)$

\section{Conclusions}

In this paper, the effects of the mass ratio, the spring constant and position, a crack and the attached mass on the stability and instability of valve-pipe system are studied by the numerical method.

As the crack severity increases, the critical flow velocity $u_{c r}$ becomes larger. When the spring position exists in the center of the pipe, the critical flow velocity has the largest value, where the crack severity is proportional to the critical flow velocity. If the crack severity is large $(\alpha>0.3)$, the re-stable region does not show up in this study. If the attached mass exists in the center of the pipe conveying fluid, the divergence unstable region appears as the fluid velocity increase. It should be remarked that the above results are presented in dimensionless form, valid for arbitrary valve-pipe system with a crack.

\section{Acknowledgment}

This work (Grants No. 00042698) was supported by Business for Cooperative R\&D between Industry, Academy, and Research Institute funded Korea Small and Medium Business Administration in 2010.

\section{References}

1. M. P. Païdoussis, Fluid-Structure Interactions (Academic Press, 1998).

2. H. I. Yoon and I. S. Son, J. Sound Vib. 292, 941 (2006).

3. T. B. Benjamin, Proc. Roy. Soc. A261, 261 (1961).

4. D. Liu, G. Gurgenci and M. Veide, J. Sound Vib. 261(1), 17 (2003).

5. M. H. F. Dado and O. Abuzeid, J. Sound Vib. 261(4), 675 (2003).

6. M. G. Kang, J. Sound Vib. 238(1), 179 (2000).

7. J. Wauer, Appl. Mech. Rev. 43(1), 13 (1990).

8. A. D. Dimarogonas, Eng. Fra. Mech. 55(5), 831 (1996).

9. I. S. Son, H. I. Yoon, S. P. Lee and D. J. Kim, Int. J. Mod. Phys. B 24(15\&16), 2609 (2010).

10. M. Kisa and J. Brandon, J. Sound Vib. 238(1), 1 (2000).

11. D. Y. Zheng and S. C. Fan, J. Sound Vib. 267, 933 (2003). 